\title{
Simulation Analysis of Tree and Mesh Topologies in Zigbee Network
}

\author{
Manpreet, Jyoteesh Malhotra \\ CSE Department \\ Guru Nanak Dev University Regional Campus \\ Jalandhar, INDIA \\ manpreetpawar17@gmail.com,jyoteesh@gmail.com
}

\begin{abstract}
Recently wireless sensor Network (WSN) and wireless personal area network (WPAN) has gained lot of interest from the research community because of its diverse applications of controlling, monitoring and automation of home, offices or any rescue area. Zigbee based on IEEE 802.15.4 standard is key enabling technology for the success of both WSN and WPAN. Zigbee has some inherent powerful characteristics like very low power consumption, localization and low cost that has drawn the attention of research community recently. Along with merits come the challenges for the success of Zigbee based networks at various layers of the network model. Network topologies in Zigbee are to be selected based on applications and performance requirements. So this paper investigates the performance of Zigbee for tree and mesh topologies. In doing so parameters like MAC throughput, MAC load, MAC delay, end to end delay have been evaluated through extensive simulations using OPNET. The intricate behavior of these topologies in Zigbee shows optimum bounds of performance for these topologies have been computed in this work
\end{abstract}

Keywords: Zigbee, OPNET, mesh topology, tree topology.

\section{Introduction}

The Zigbee wireless technology, protocol developed for short range communications using radio frequency has powerful features such as low data rate, low power consumption, security, and reliability with low cost. It is efficiently contributing in WPAN and WSN. It is the enhancement of IEEE 802.15.4 which has only two layers i.e. physical and MAC layer [1]. The layered architecture of Zigbee has 4 layers: physical layer, MAC layer, network layer and application layer. The Zigbee protocol supports static, dynamic, and meshes network topologies. In order to handle defect caused by various environmental effects, the Zigbee protocols offer self-healing capability for the network to sense and recover from network or communication link faults without human interference. Zigbee states three frequency bands supporting different channels: $2.4 \mathrm{GHz}$ band afford 16 channels, 902-928 $\mathrm{MHz}$ band afford 10 channels and $868-870 \mathrm{MHz}$ band afford 1 channel. The maximum limit of data rate for each band is $250 \mathrm{kbps}, 40 \mathrm{kbps}$ and $20 \mathrm{kbps}$ respectively [2]. It supports direct spread spectrum for shared communication. Power life of Zigbee network is 100 to 1000 days. Apart from star, tree and mesh, multiple hybrid topologies can be developed in the Zigbee protocols which are combination of these basic topologies. The evaluation of optimum performance in respect of these basic topologies will be essential for understanding the behavior of Zigbee protocols. Various researchers shows the behavior of star, mesh and tree topologies for various parameters [4] [6] [7] and this paper shows the results of tree 
and mesh topologies for different parameters using different power reception value and shows the self-healing behavior of mesh topology.

The rest of paper is organized as follows. Next section gives the brief introduction of the layered architecture of Zigbee. In section 3 network topologies have been presented. The simulation results and discussion have been done in section 4 . Section 5 provides the conclusion and future scope of work done.

\section{Layered Architecture}

Zigbee consists of four layers. The top two (Application and Network) layers are specified by the Zigbee Alliance and the bottom two (Medium Access Control and Physical) layers specified by the IEEE 802.15.4-2006 [3].

\subsection{Application Layer}

Application layer is provided by the Zigbee Alliance. It is the top most layers. It is very close to end user and very useful for user. All applications of Zigbee run on this layer. For example, applications to monitor temperature, humidity, or any other desirable atmospheric parameters can be placed on this layer for agricultural use.

2.1.1 Zigbee Device Object (ZDO): ZDO is a special type application run on every Zigbee device. This application defines the type of Zigbee device (end device, router, and coordinator) a particular node is, initializing the network, and to also participate in forming a network.

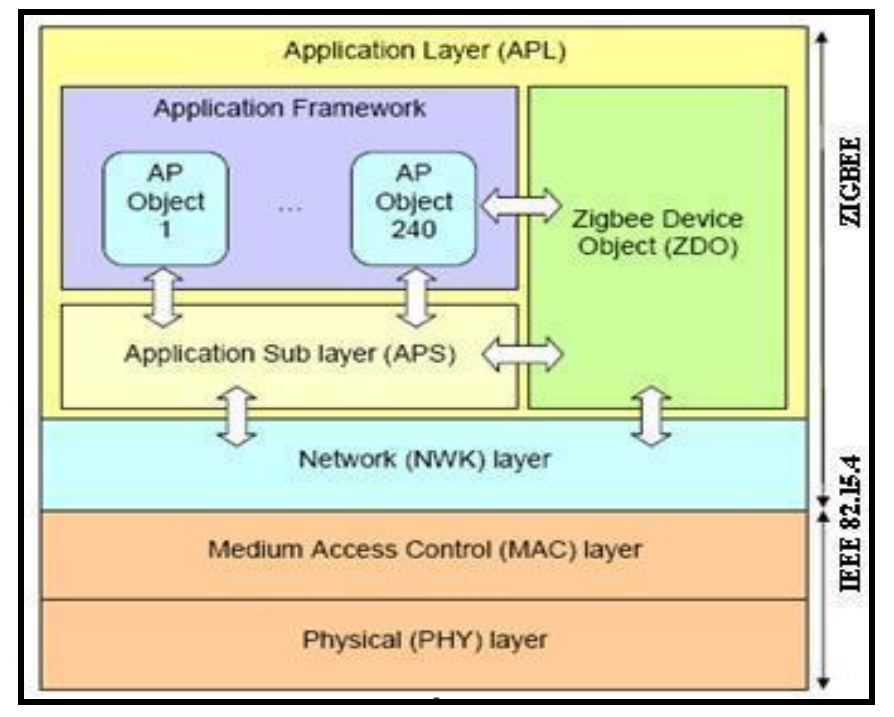

\section{Figure 1. Layered architecture of Zigbee [9]}

\subsection{Network Layer}

MAC layer is provided by the Zigbee Alliance. This layer provide interface between application layer and MAC Layer. This layer provides routing, network management, security management etc. Self healing which is a powerful feature of Zigbee is also provided by this layer. This layer is defined by the Zigbee Alliance, which is an association of companies united to work for a better Zigbee standard. 


\subsection{Medium Access Control Sub-layer}

MAC layer is provided by the IEEE 802.15.4 standard. It provide interface between MAC Layer and physical layer. It accomplishes addressing of data to determine from where the frame is departing, or where it going to arrive. It is also responsible for multiple access control such as CSMA/CA permit for unfailing convey of data and beaconing.

\subsection{Physical Layer}

The physical layer is provided by the IEEE 802.15 .4 standard. It is very close to hardware. This standard deals with the physical transmission of radio waves in different unlicensed frequency bands around the world to provide communication between devices within a WPAN. These frequency bands support different channels: $2.4 \mathrm{GHz}$ band provide 16 channels with $250 \mathrm{kbps}$ data rate, 902-928 $\mathrm{MHz}$ band provide 10 channels with $40 \mathrm{kbps}$ data rate and $868-870 \mathrm{MHz}$ band provide 1 channel with $20 \mathrm{kbps}$ data rate. It offers two modulation key: Binary phase shift key (BPSK) and Q-OPSK (quadratic offset phase shift key). This layer selects channel to avoid radio interference, as well as data exchange with the layer above (MAC sub-layer) it.

\section{Zigbee Devices and Network Topologies}

A Zigbee network is a mixture of three basic components- a Zigbee coordinator, a Zigbee router, and a Zigbee end device. These components and Topologies are briefly defined below.

\subsection{Zigbee Devices}

3.1.1. Coordinator: It starts the network, choose appropriate channel \& link up other devices in the network. End devices or routers can directly connect with this device. Other both devices can directly communicate with coordinator. It allocates time slots to other devices in time critical application.

3.1.2. Router: It relays message between devices. It keeps the back up of routes. In case of failure, router recovers the route .It enhances the network coverage. It provides interface between coordinator and end devices.

3.1.3. End Device: It works as source and sink for messages. Mostly it remains in sleep mode and saved battery. It can communicate with both other devices in active mode.

\subsection{Network Topologies}

Performance of Zigbee network depends upon the topology formation support. Basically it supports three topologies: Star, Tree and Mesh. But by combining these topologies, multiple hybrid topologies can be made. Topology structure dependence upon the situation of devices: Coordinator, Router and end devices.

3.2.1. Star Topology: In star topology Zigbee coordinator in surrounded by devices as shown in figure 2.a. It can be easily configured. It can support up to 6000 devices. But there are also some limitations: If coordinator failed to work due to some technical liability then whole network fails because all traffic go through the coordinator of the star so this topology does not offer reliable transmission. 


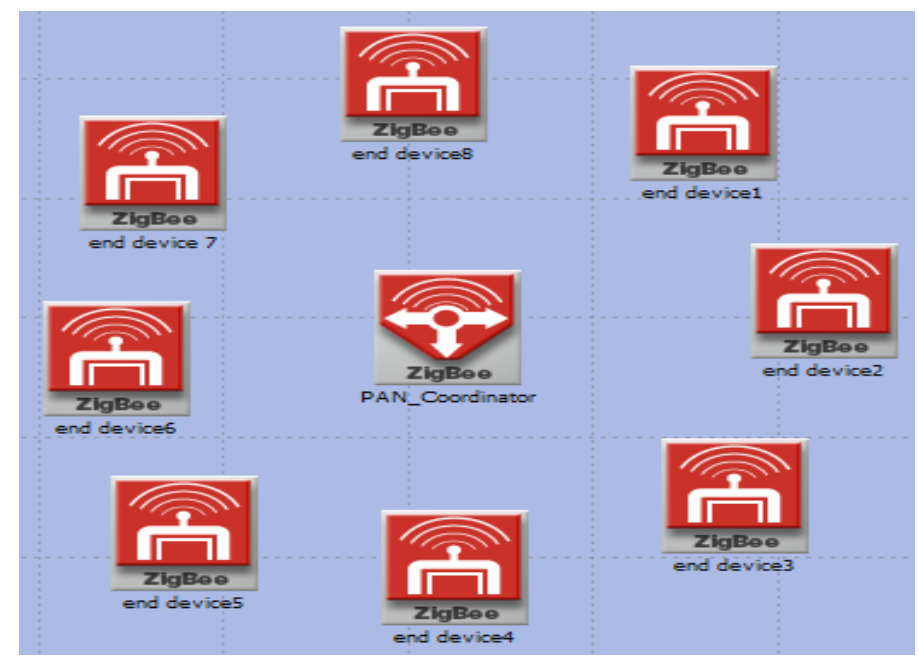

Figure 2.a Star Topology

3.2.2. Tree Topology: In tree topology Zigbee Coordinator positioned at the root of the network. Other devices except can connect to coordinator and number of end devices can connect to routers as children. Only routers and coordinator can act as parent nodes because end device have no capability to relay message. When any node want to transmit message to other node then it send message to its parent node which is one level higher than it then that message is relayed higher until it reach to its destination. If any router fails to work then its children (end devices) also stop working.

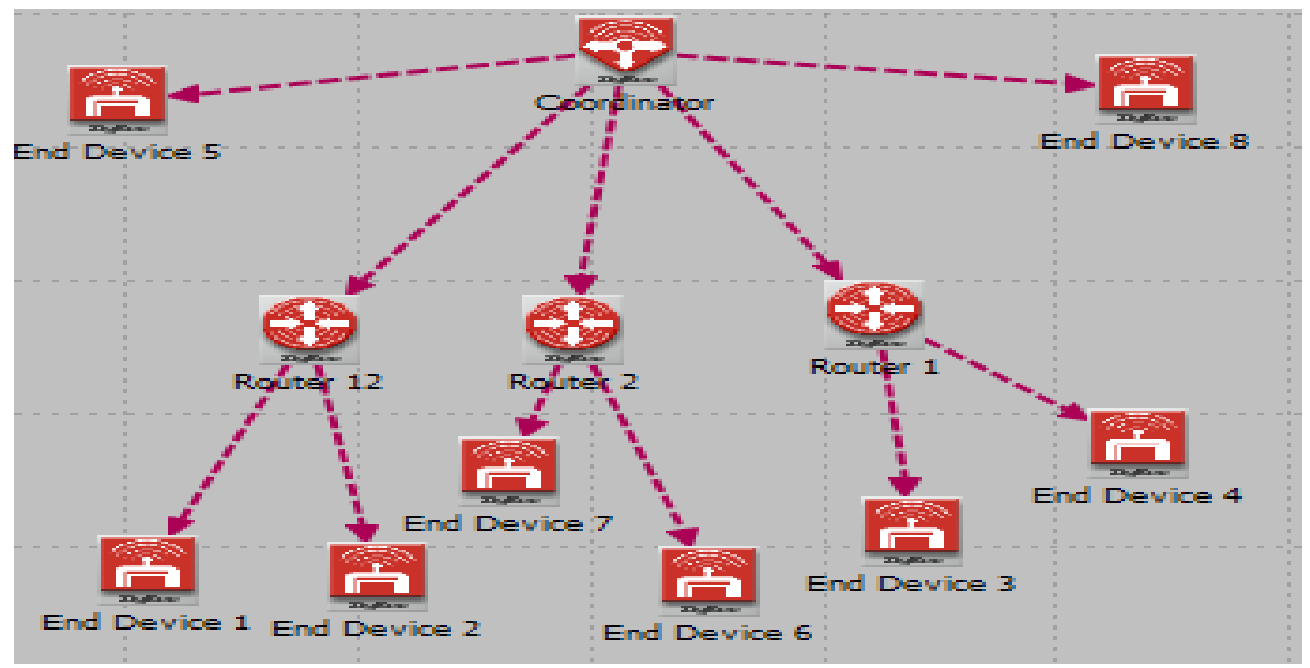

Figure 2.b Tree Topology

3.2.3. Mesh Topology: This is the most flexible and trustworthy topology because there are number of potential paths for a message so if any router fails to work then Zigbee's self healing mechanism search other path and message can be relay through other path. There are number of alternate paths. 


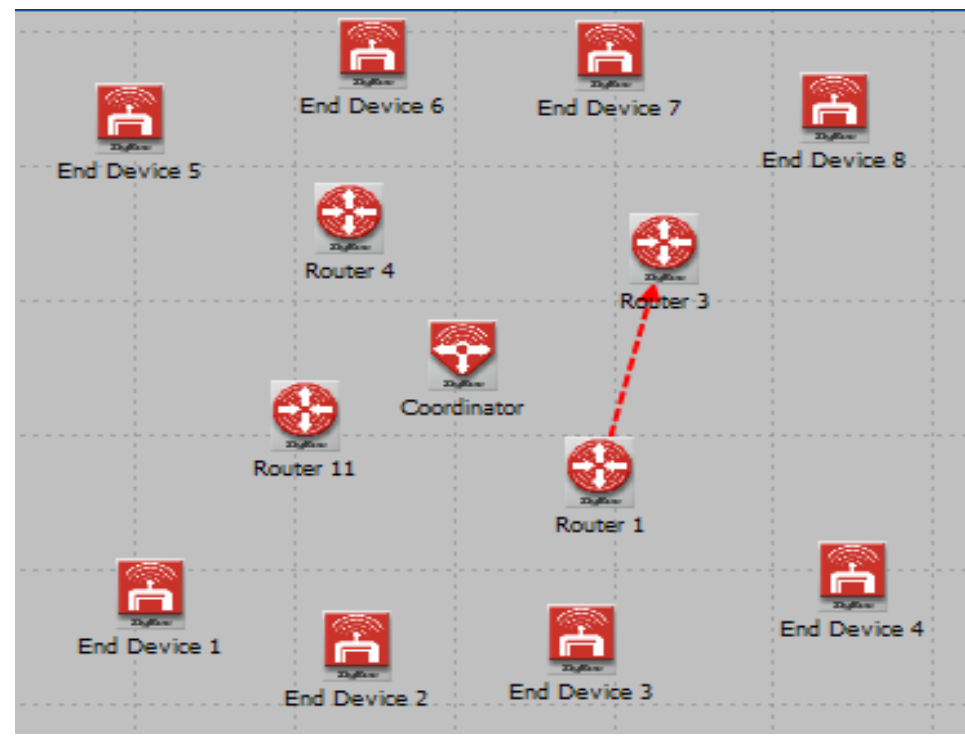

Figure 2.c Mesh Topology

\section{Simulation Results and Discussion}

In this section, the simulative investigations of different topologies have been done to find out the optimum behavior of topologies. OPNET provides a Virtual Network Environment that models the behavior of entire network, including its routers, switches, protocols, servers, and individual applications. In this paper, study of the intricate behavior of mesh and tree topologies in Zigbee networks through realistic channel conditions has been done. In [8] role of simulation software OPNET in Simulation of Topologies of Zigbee Network has been valued.

\subsection{Scenario1: Mesh Topology Analysis by Varying the Packet Reception and Power Threshold Values and Reducing the Transmit Power}

In this scenario, the behavior of mesh topology is analyzed by varying the packet reception and power threshold value which was initially set at default value of -85 $\mathrm{dBm}$. Packet reception defines the receiver sensitivity in $\mathrm{dBm}$. Packets whose reception power is less than the threshold will not be sensed by the MAC. In this scenario mesh topology network has been created and set the transmission band to Worldwide, enabled ACK mechanism . The default transmit power was $0.05 \mathrm{~W}$ and packet reception power threshold was at $-85 \mathrm{Dbm}$. Transmit power is reduced to half i.e $0.025 \mathrm{~W}$ and considered following three values for packet reception:-

$1.76 \mathrm{dBm}$

$2.80 \mathrm{dBm}$

$3.90 \mathrm{dBm}$

Then the network simulated for 10 minutes and obtained the results given below. In the results the blue line is indicates data at $-85 \mathrm{dbm}$, yellow for $-80 \mathrm{dBm}$, green for $-76 \mathrm{dBm}$ and light blue for $-90 \mathrm{dBm}$.

4.1.1. Throughput: Throughput means the number of successful transmissions. It is observe from the Figure 3 that by changing the packet reception to $-76 \mathrm{dBm}$ (red line) the throughput is less (between 14,000-12,000 dBm), at $80 \mathrm{dBm}$ (green line) the throughput is irregular as continuous peaks and falls are observed, at $85 \mathrm{dBm}$ (blue line) it's approximately $14,000 \mathrm{bits} / \mathrm{sec}$. At $-90 \mathrm{dBm}$ and above from $-90 \mathrm{sBm}$ the throughput does not rises but remains the same as at $-85 \mathrm{dBm}$. 


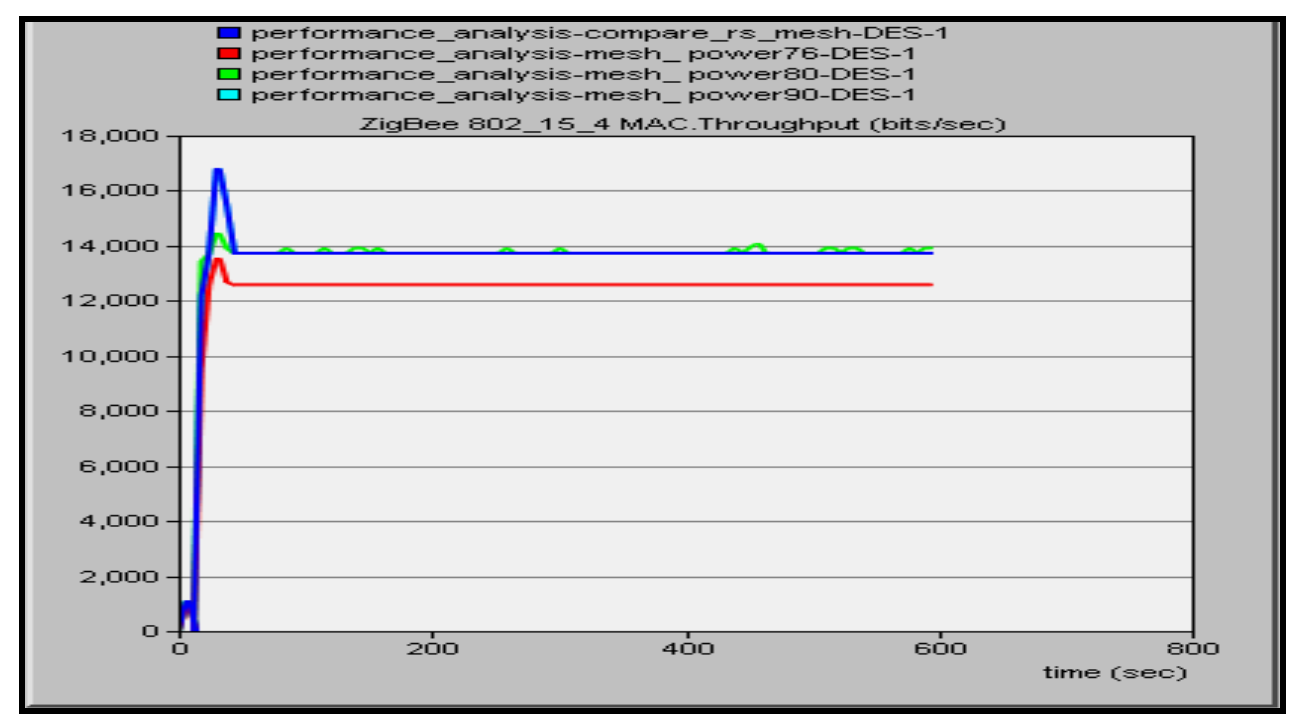

Figure 3. MAC Throughput (bits/sec)

4.1.2. Load: The load is affected only when power reception is changed to -76 $\mathrm{dBm}$ from the default value of $-85 \mathrm{dBm}$. For all other values it is the same as that at $-85 \mathrm{dbm}$ as observed form figure 4 .

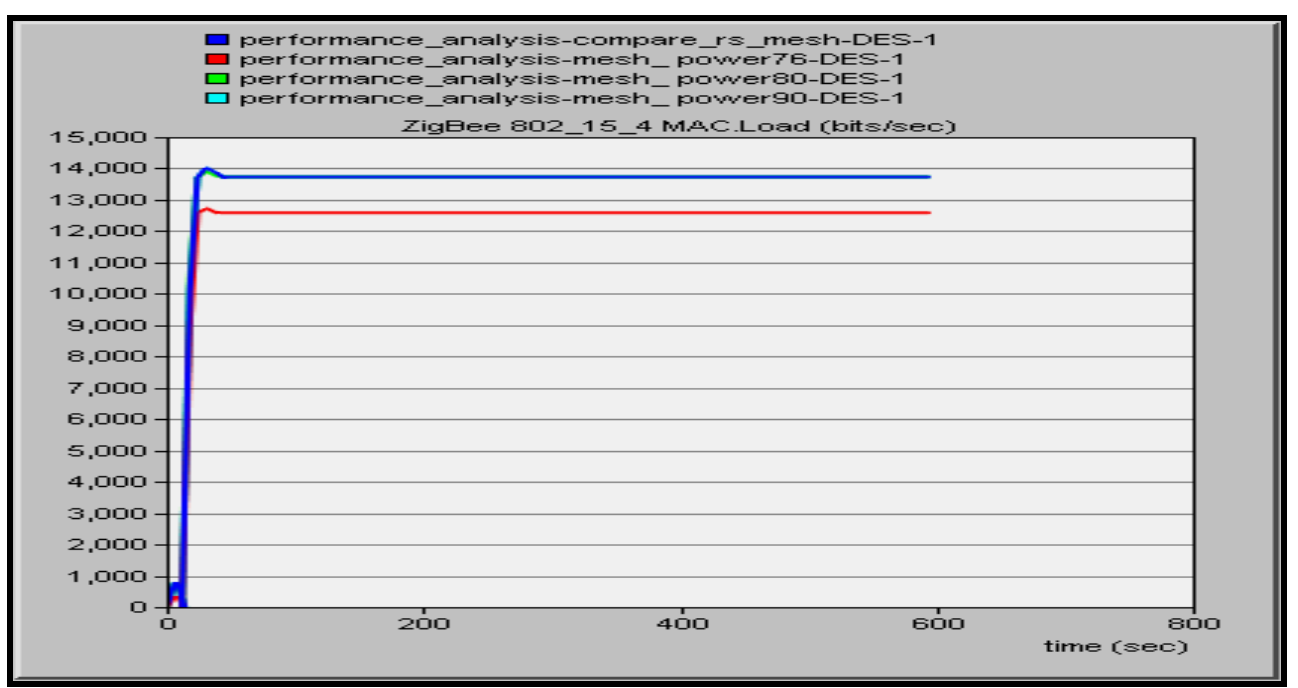

Figure 4. MAC Load (bits/sec)

4.1.3. Data Traffic Received: Data traffic received is minimum when packet reception is at $-76 \mathrm{dBm}$. At $-90 \mathrm{dBm}$ there is no variation from the default value. At $-80 \mathrm{dbm}$ the graph contains curves many small peaks so variation is slightly irregular as shown in figure 5 . 


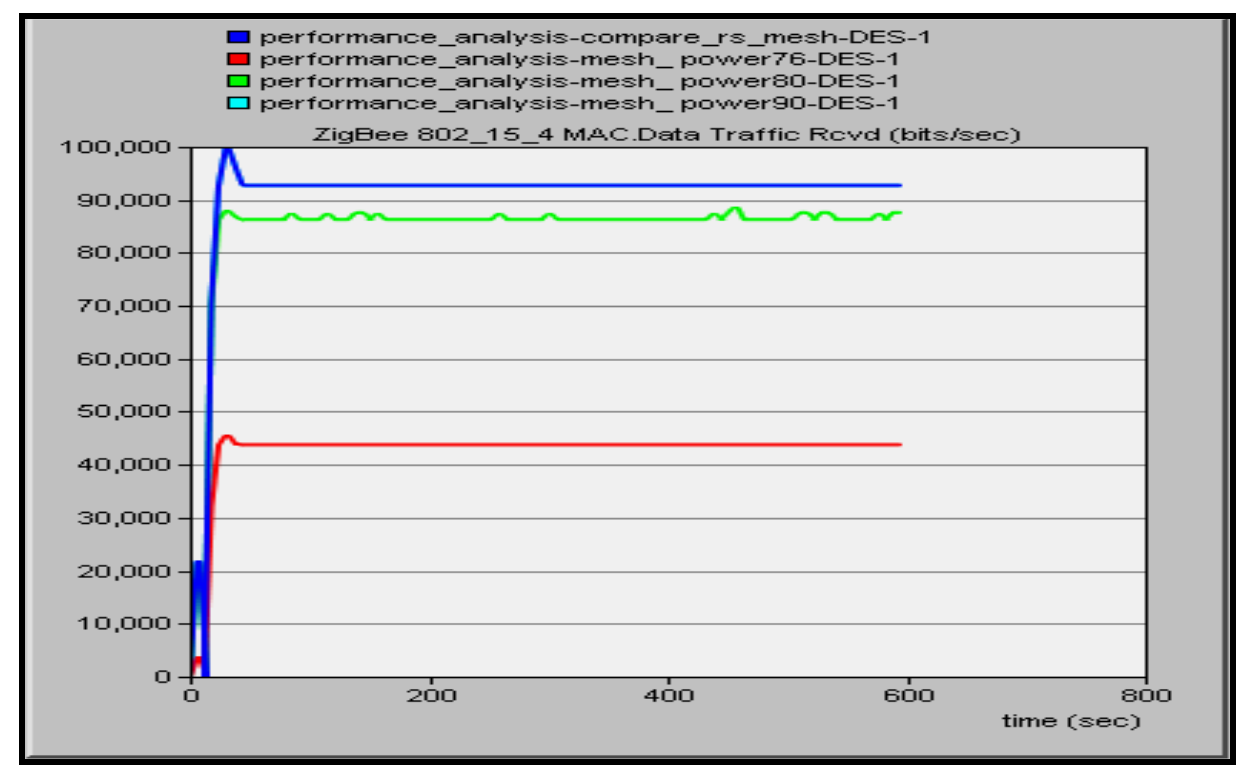

Figure 5. Data Traffic Received (bits/sec)

4.1.4. Delay: Delay almost the same in all the cases with very small variations. Initially the delay is high because of the time taken by nodes to find the paths for transmission. For $-76 \mathrm{dBm}$, the delay is $0.01 \mathrm{sec}$ and for others it lies between 0.010.02 secs as shown in figure 6 .

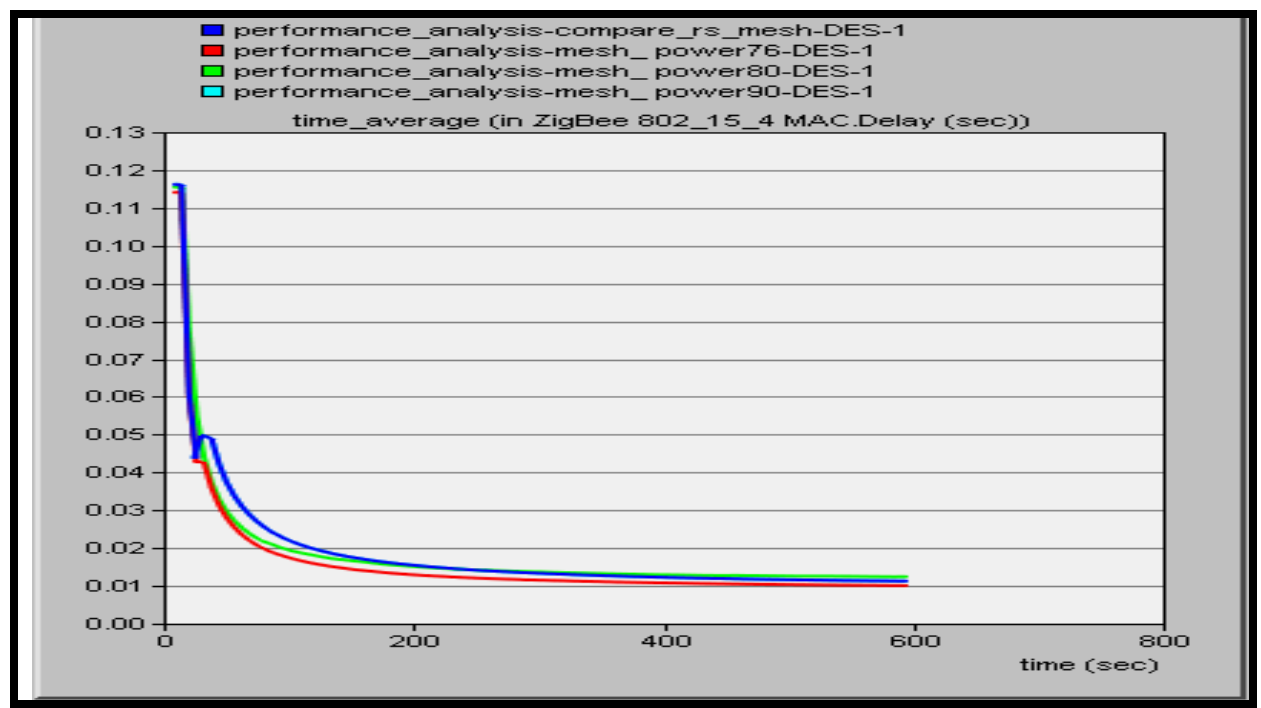

Figure 6. MAC Delay (sec)

4.1.5. End to End delay: End to end delay is maximum at $-80 \mathrm{dBm}$ and minimum at $-76 \mathrm{dbm}$. At $-85 \mathrm{dBm}$ and $-90 \mathrm{dBm}$, the value lies intermediate of the two as shown in figure 7 . 


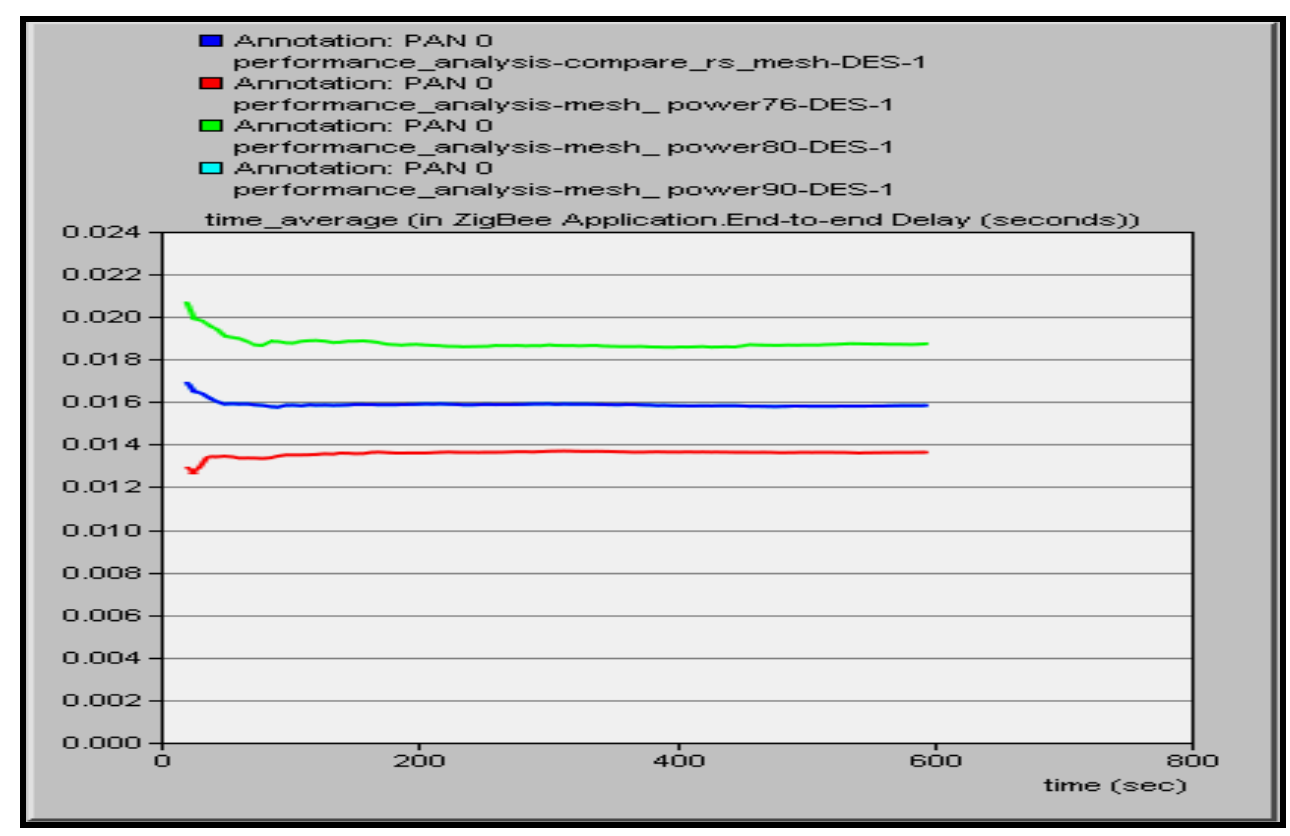

Figure 7. End to End Delay (Seconds)

4.1.6. Results: Results show that by setting the packet reception and power threshold to $-76 \mathrm{dbm}$ the performance of mesh topology deteriorates as there is fall in throughput, load and data traffic received. At $-80 \mathrm{dBm}$, the performance is good but not regular as observed the small variations in the curves occurring frequently. Below $-85 \mathrm{dBm}$, that is at $-90 \mathrm{dBm}$ there is no change observed. So for values below $-85 \mathrm{dBm}$ the performance is not affected.

\subsection{Scenario 2: Studying the Behavior of Tree and Mesh Topologies when the Router Fails}

In this scenario Zigbee network is simulated, and studied the self healing mechanism in mesh topology. Comparison of the performance of the tree and mesh topologies is done. The scenario consists of 1 coordinator, 2 routers and 7 end devices. Each node has its PAN id set to auto assigned. Every node has its destination set to random. One scenario with tree topology and other with mesh topology are created. To study router failure condition, a Failure recovery device in the workspace is placed. Router1 fails after 120 seconds and Router2 after 200 seconds. The Router 1 recovers after 360 seconds from failure and Router 2 recovers after300 seconds from failure. Scenario1 name is for tree topology network and mesh for the mesh topology network. This case is analogous to the situation when the router is blown away in the agricultural fields due to winds.

4.2.1. Throughput: In figure 8 the Blue line indicates the Mesh topology and the red line indicates tree topology. It is observed that during the failure of the Router 1 after 120 seconds, the throughput of both the tree and mesh networks fall. It further falls when the Router 2 fails after 200 seconds. Then with the recovery of both the routers the throughput rises. On the whole, Mesh Topology is better as the number of successful transmissions is more in the Mesh network. It is $22,000 \mathrm{bits} / \mathrm{sec}$ in Mesh and attains a value of $11,000 \mathrm{bits} / \mathrm{sec}$ after nearly 420 secs. 


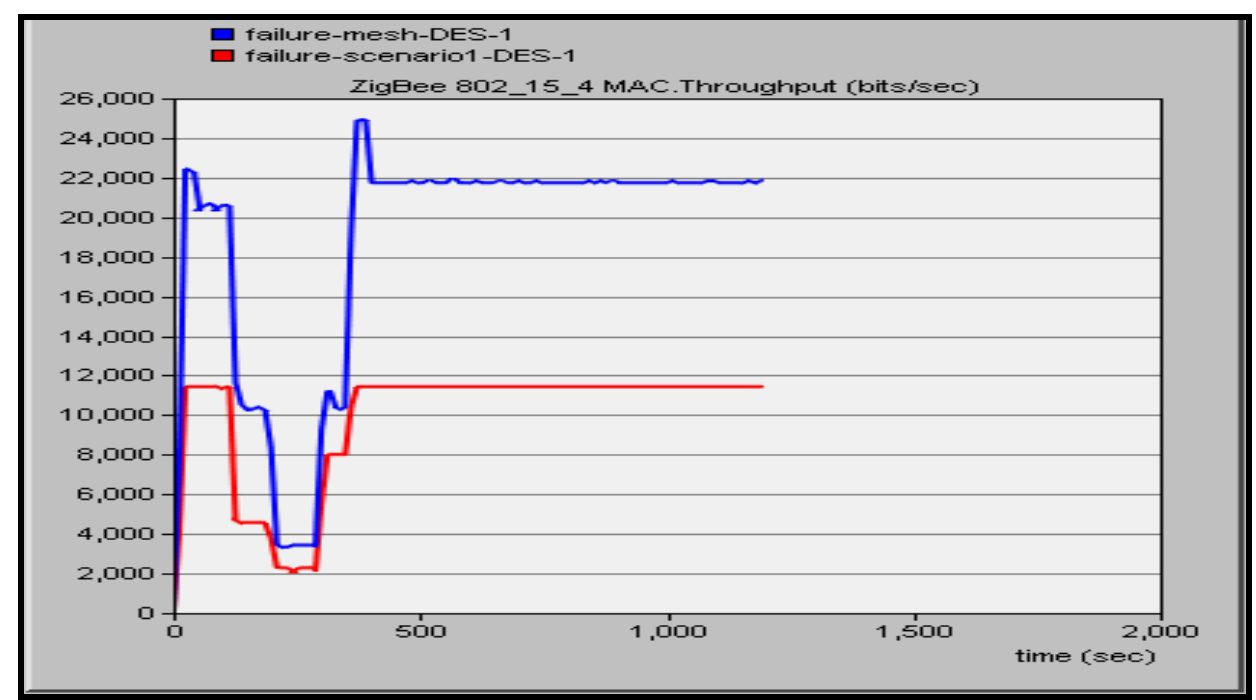

Figure 8. MAC Throughput (bits/sec)

4.2.2. Delay: Delay decreases with the time in both the networks as observed from the Figure 9. But delay of Mesh is more as compared to Tree topology. The initial rise (blue line) in the delay for mesh topology is due to the time spent in the management and control traffic transmitted by the devices to perform the route discovery and after that delay falls. The peak and irregularities observed in delay of mesh are due to failure of router and the time taken by nodes to find an alternative path.

Figure 10 shows that due to the self healing mechanism, sharp peaks for the intervals are observed when the routers fail. Tree topology shows zero delay for these intervals which means no data is reaching the coordinator whereas in mesh due to self healing, transmissions occur but delay increases because the nodes have to find an alternative path. Hence the sharp rise in delay.

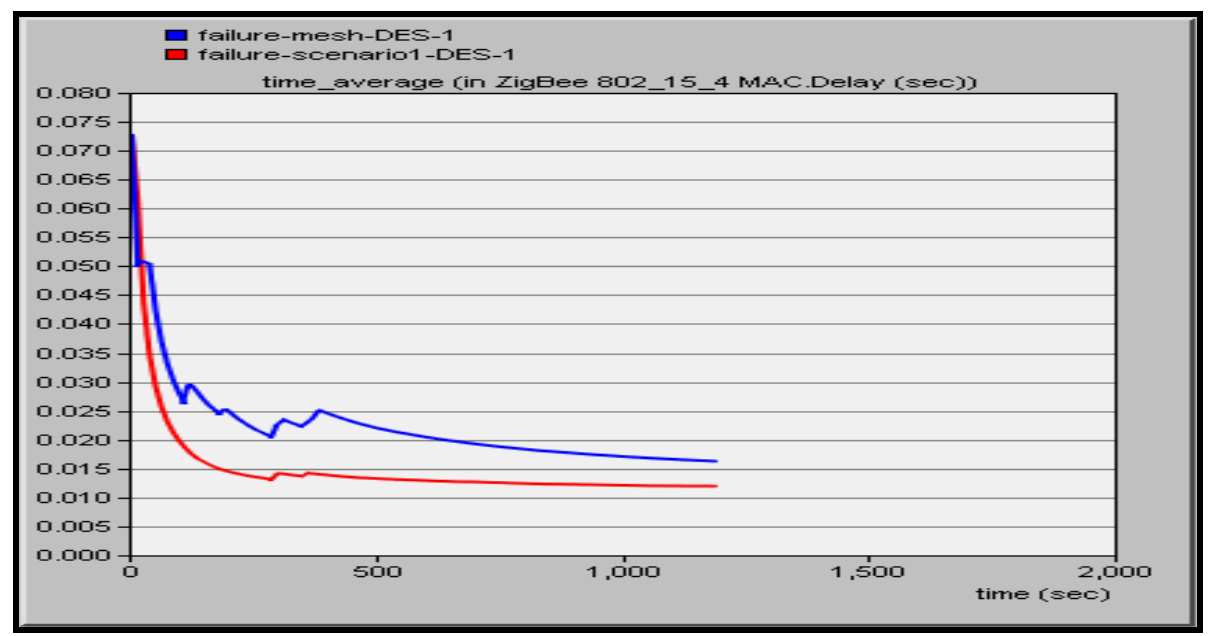

Figure 9. MAC Delay (sec) 


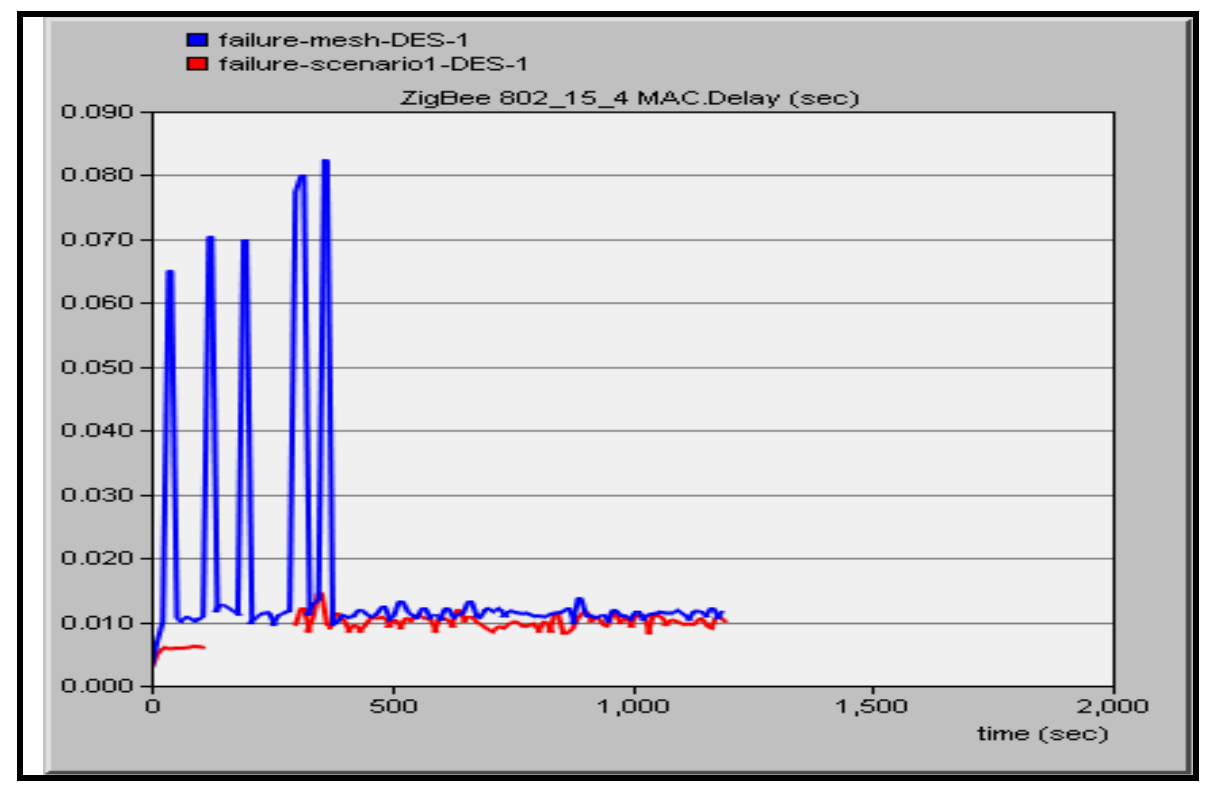

Figure 10. MAC Delay(Sec)

4.2.3. Load: From Figure 11 the self healing feature of the Mesh network is observed. It is observed that in mesh topology, even though Router 1 fails, the load is not much affected. This is because the nodes find an alternative path to transmit data. Hence the transmission of data continues. This feature of mesh topology is called self healing, which is not observed in tree topology. This explains the graph shown below.

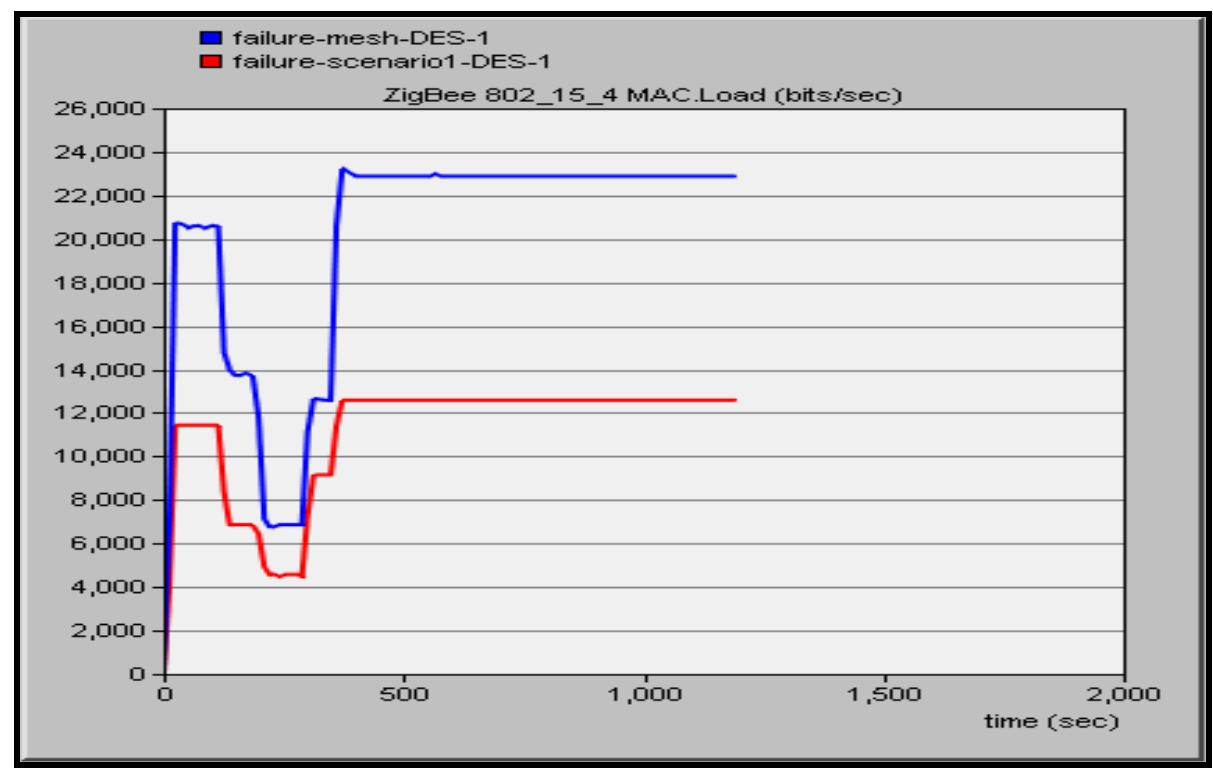

Figure 11. MAC Load (bits/sec)

Observing Figure 12, the self healing mechanism exhibited in mesh topology is seen. With the failure of Router1 after 120 seconds, still the load of coordinator remains the same i.e. the total no. of transmissions of the network reaching the coordinator remains the same. There are slight irregularities observed in form of small downward peaks but those are due to the management and control traffic sent 
by the nodes to find an alternative path for transmission. Hence self healing is observed and no such feature is found in Tree topology.

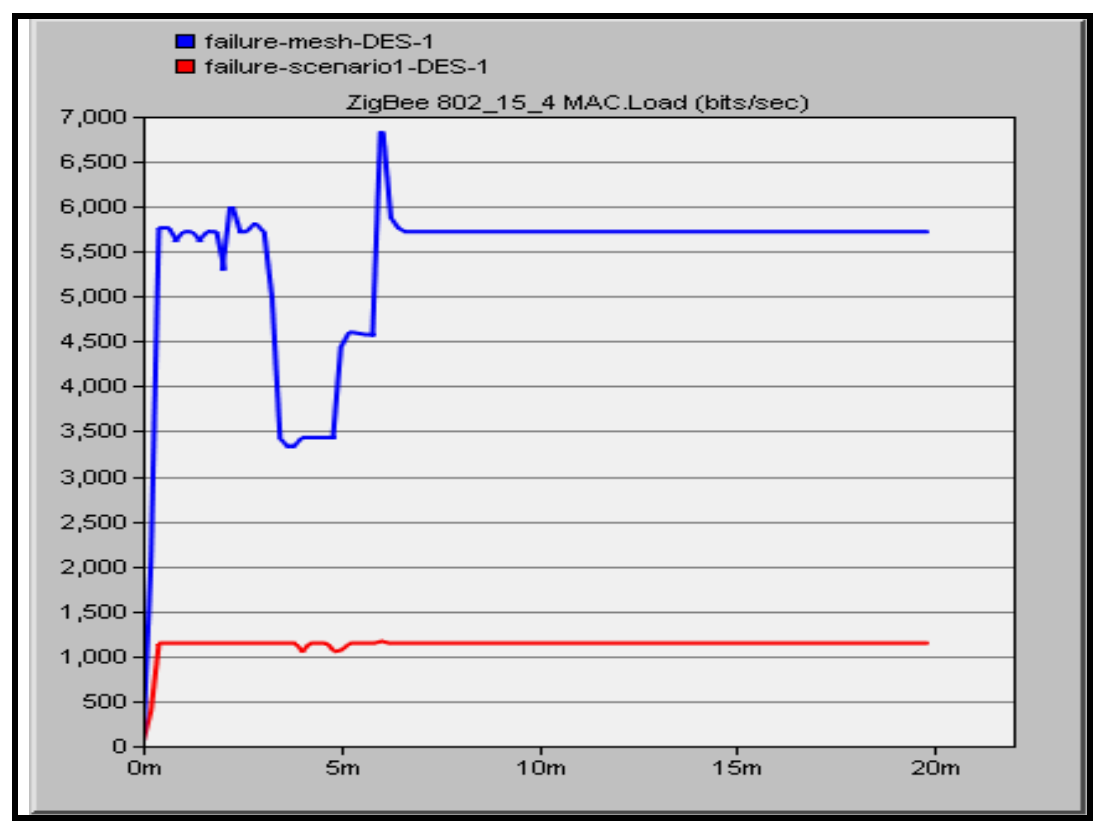

Figure 12. MAC Load (bits/sec)

4.2.5. Result: The self healing mechanism is observed in mesh networks as all the nodes are interconnected in the mesh topology and tree networks do not have any such feature. This feature helps mesh networks to maintain a consistent performance and due to this feature the nodes are able to find an alternative path for transmitting data when the failure occurs.

\section{Conclusion and Future Scope}

In above scenarios, intricate behavior of the topologies using OPNET has been studied. The whole performance of first scenario shows that only at $-85 \mathrm{dBm}$ the performance is optimum and second scenario concluded that mesh is more reliable but its cost is high. So mesh topology can be preferred in the situations where cost is no restriction like in defense applications or military applications. Otherwise tree topology can be used for the consistent performance with the limitation that fault can't be self healed and it's less reliable.

In future we will study the behavior of mesh and tree topologies by taking parameters like back off exponent, increasing the number of nodes, creating small, medium and large sized Zigbee networks and failing the coordinator. The work done in this paper indicates the significant role of OPNET in studying the wireless networks.

\section{References}

[1] Zigbee Alliance, Zigbee Overview, (2003).

[2] J. Shyan Lee, Y. W. Su and C. C. Shen, "A Comparative Study of Wireless Protocols:Bluetooth, UWB, Zigbee, and Wi-Fi", The 33rd Annual Conference of IEEE Industrial Electronics Society (IECON), (2007); Taipei, Taiwan.

[3] R. C. Muthu, M. Shanmugaraj and R. Prabakaran, "Study on Zigbee Technology", 3rd International Conference on Electronics Computer Technology (ICECT), IEEE, (2011).

[4] B. Mihajlov and M. Bogdanoski, "Overview and analysis of the performance of Zigbee based wireless sensor networks", International Journal of Computer Applocation, vol. 29, no. 12, (2011). 
[5] M. Dharmistha and D. Vishwakarma, "IEEE 802.15.4 and Zigbee: A Conceptual Study", International Journal of Advanced Research in Computer and Communication Engineering, vol. 1, (2012), no. 7.

[6] M. Hammoshi and A. Sayed, "An analysis for a cluster tree Zigbee topology", Journal of Theoretical and Applied Information Technology, vol. 64, no. 3, (2014).

[7] K. Vats, P. Jain, L. Jaiswal and S. Singh, "Zigbee based WSN topology Simulation Investigation and performance analysis using OPNET", International Journal of Advanced Scientific Research and Technology, vol. 3, no. 2, (2012).

[8] I. S. Hammoodi, B. G. Stewart, A. Kocian and S. G. McMeekin ,"A Comprehensive Performance Study of OPNET Modeler For Zigbee Wireless Sensor Networks", IEEE Third International Conference on Next Generation Mobile Applications, Services and Technologies, (2009).

[9] L. Jaiswal, J. Kaur and G. Singh, "Performance Analysis of Topological Variation in Personal Area Network using Zigbee Wireless Sensors", International Journal of Computer Science And Technology(IJCST), vol. 3, no. 4, (2012).

[10] A. Kaur, J. Kaur and G. Singh, "An Efficient Hybrid Topology Construction in Zigbee Sensor Network", IEEE International Conference on Recent Advances and Innovations in Engineering (ICRAIE), (2014); Jaipur, India. 